Pacific Journal of Mathematics

MANIFESTLY DYNAMIC FORMS IN THE CARTAN-HAMILTON 


\title{
MANIFESTLY DYNAMIC FORMS IN THE CARTAN-HAMILTON TREATMENT OF CLASSICAL FIELDS
}

\author{
RICHRD ARENS
}

\begin{abstract}
Our intent is to show that certain differential forms, which are manifestly closed on the motions of a classical field system, are Hamiltonic in the sense of generating a canonical vector field, or are equivalent to Hamiltonic forms.
\end{abstract}

1. Introduction. Let $K$ be a differentiable manifold of dimension at least $m$ and let $\alpha$ be a differential form of degree $m$ defined in $K$. Familiar considerations from the calculus of variations then leads to certain $m$-dimensional submanifolds of $K$, the extremals for $\alpha[1]$.

For $m=1$ this reduces to the Hamiltonian formalism of dynamics, and in this case a dynamic variable is a function on $K$ which is constant on the extremals. The generalization to $m \geqq 1$ is to define an $(m-1)$-form $\varphi$ to be a dynamic form for $\alpha$ if the restriction $d \varphi \mid E$ of its differential to each extremal $E$ vanishes $[1,2,3,4,5$, $6,7,8]$.

A special class of dynamic forms are the Hamiltonic forms [4] $[7,111]$. An $(m-1)$-form $\varphi$ in $K$ is Hamiltonic if there is a vector field $U$ on $K$ such that $d \varphi$ coincides with the interior product $U\lrcorner d \alpha$. For $\varphi$ Hamiltonic as above and $\psi$ any $(m-1)$-form, a new $(m-1)$-form

$$
\{\varphi, \psi\}=U\lrcorner d \psi
$$

has been defined in [4], and called the Poisson bracket.

In the theory of Kijowski, the dynamic forms whose support has a certain compactness property $[6,112]$ are used to define observables by being integrated over extremals. Two dynamic forms whose difference vanishes on every extremal are called equivalent.

Our intent is to give a sufficient condition that a form be equivalent to a Hamiltonic form. The condition ("manifestly dynamic form") does not involve the postulation of a canonic vector field $U$. The precise definitions require a preliminary discussion of the type of $m$-form $\alpha$ to be considered.

The $m$-form $\alpha$ is required to originate in a Lagrangian [4]. The $\alpha$ is supposed to be defined on a first-order jet bundle

$$
J^{(1)}\left(Q, R^{m}\right)
$$


where $Q$ is some differentiable manifold. (The integer $n$ is reserved for the dimension of $Q$. When $m=1, Q$ is the configuration space. Otherwise it can be regarded as the range of a field over spacetime.)

Such an $m$-form is expressible by a formula which we take from $[4,(3.15)]$, with a change of notation:

$$
\alpha=\sum_{i=1}^{m} \sum_{\lambda=1}^{n}(-1)^{i+1} p_{\lambda}^{i} d x^{\lambda} d t^{1} \cdots d t^{i-1} d t^{i+1} \cdots d t^{m}-H d t^{1} \cdots d t^{m} .
$$

Although intending the Grassmann product here, we will omit the $\operatorname{sign}(\wedge)$ employed in [4].

2. Some formal properties of $\alpha$ and $d \alpha$. Suppose $(i, j, \cdots, k)$ is an ordered $m$-tuple of integers $1 \leqq i, j, \cdots, k \leqq m$. Let

$$
E_{i j \cdots k}=0 \quad \text { if they are not all distinct }
$$

and

$$
E_{i j \cdots}= \pm 1 /(m-1) !
$$

when they are an even (or odd, respectively) permutation of $1,2, \cdots, m$. Then we can use the summation convention and write

$$
\alpha=E_{i j \ldots k} p_{\lambda}^{i} d x^{\lambda} d t^{j} \cdots d t^{k}-H d t^{1} \cdots d t^{m} .
$$

Repeated Roman indices are summed 1 to $m$ and Greek indices, 1 to $n$. Thus

$$
d \alpha=E_{i j, \cdots, k} d p_{\lambda}^{i} d x^{\lambda} d t^{j} \cdots d t^{k}-d H d t^{1} \cdots d t^{m} .
$$

Let us introduce some 1-forms

$$
X^{\lambda}=d x^{\lambda}-\frac{\partial H}{\partial p_{\lambda}^{i}} d t^{i},
$$

and some $m$-forms

$$
P_{\lambda}=E_{i j, \cdots, k} d p_{\lambda}^{i} d t^{j} \cdots d t^{k}+\frac{\partial H}{\partial x^{\lambda}} d t^{1} \cdots d t^{m} .
$$

The vanishing of these forms is the content of Hamilton's canonic equations when $m=1$.

THEOREM.

$$
\begin{gathered}
d \alpha=-X^{\lambda} P_{\lambda} . \\
\left.\frac{\partial}{\partial x^{\lambda}}\right\rfloor d \alpha=-P_{\lambda} .
\end{gathered}
$$




$$
\left.\frac{\partial}{\partial p_{\lambda}^{i}}\right\rfloor d \alpha=X^{\lambda} E_{i j \cdots k} d t^{j} \cdots d t^{k} .
$$

These are elementary consequences of 2.1 and 2.2. From 2.5 one has the following:

THEOREM 2.6. Let $A_{1}, \cdots, A_{n}$ be $(m-1)$-forms involving only $d t^{1}, \cdots, d t^{m}$. Then there exists a vector field $U$ such that

$$
U\lrcorner d \alpha=X^{\lambda} A_{\lambda} .
$$

A property characterizing extremals $E$ for $\alpha$ is this: $E$ is an $m$-dimensional submanifold and whenever $U$ is a vector then the restriction $(U\lrcorner d \alpha) \mid E$ is 0 [4], [8, (1.1)].

THEOREM. An m-dimensional submanifold $E$ is an extremal for $\alpha$ if

$$
X^{\lambda} \mid E \text { and } P_{\lambda} \mid E \text { are } 0 \quad \text { for } \lambda=1, \cdots, n \text {. }
$$

Proof. $\left.\left.(U\lrcorner d \alpha) \mid E=(U\lrcorner X^{\lambda}\right)\left|E \wedge P_{\lambda}-X^{\lambda}\right| E_{\wedge}(U\lrcorner P_{\lambda}\right) \mid E=0$.

An extremal $E$ shall be called a motion if the forms $\left(E_{i j \cdots_{k}} d t^{j}, \cdots d t^{k}\right) \mid E$ are not all 0 at any point (of $\left.E\right)$. The extremals met in applications are invariably motions. In fact $d t^{1} \cdots d t^{m}$ isn't 0 on them; moreover, every extremal is a motion when $m=1$.

THEOREM. Let $E$ be a motion. Then 2.7 holds.

Proof. We can infer that the forms in 2.4 and 2.5 vanish on $E$. Therefore $P_{\lambda} \mid E=0$, while 2.5 and regularity yield $X^{\lambda} \mid E=0$.

A different sort of property of $\alpha$ is this.

Proposition. Suppose that

$$
\frac{\partial H}{\partial t^{m}}=0
$$

Then

$$
\left.\frac{\partial}{\partial t^{m}}\right\lrcorner \alpha
$$

has, apart from a constant factor, just the form 1.1 with $m$ diminished by 1.

This form 2.9 is Hamiltonic, when 2.8 holds. 
3. Manifestly dynamic forms. Any $m$-form which can be written as

$$
A_{\lambda} X^{\lambda}+B_{\lambda} d X^{\lambda}+c^{\lambda} P_{\lambda}
$$

(where these coefficient-forms are of degree $m-1, m-2$, and 0 respectively) vanishes when restricted to any motion.

If $\varphi$ is an $(m-1)$-form where $d \varphi$ is of the form 3.1 then the restriction $d \varphi \mid E$ to any motion $E$ is 0 and thus $\varphi$ is manifestly dynamic. We will define $\varphi$ to be manifestly dynamic precisely when $d \varphi$ is of the form 3.1.

Proposition. [8, 112] A Hamiltonic form is manifestly dynamic.

Proof. If $d \varphi=U\lrcorner d \varphi$ then $\left.\left.d \varphi=-(U\lrcorner X^{\lambda}\right) P_{\lambda}+X^{\lambda}(U\lrcorner P_{\lambda}\right)$, which is of the form 3.1. The converse is discussed in $\S 6$.

Our first theorem is of a local kind.

THEOREM 3.2. Let $\varphi$ be manifestly dynamic in the neighborhood of some point. Then in some neighborhood of that point there exist $(m-2)$-forms $F_{1}, \cdots, F_{n}$ such that $\varphi-F_{\lambda} X^{\lambda}$ is Hamiltonic.

Proof. We call any form like $F_{\lambda} X^{\lambda}$ an $X$-form. Our method is to keep subtracting $X$-forms from $\varphi$ until we come to a Hamiltonic form.

We are given that $d \varphi=A_{\lambda} X^{\lambda}+B_{\lambda} d X^{\lambda}+c^{\lambda} P_{\lambda}$.

Clearly $d\left(\varphi+B_{\lambda} X^{\lambda}\right)$ is of the form $C_{\lambda} X^{\lambda}+c^{\lambda} P_{\lambda}$. In other words, we have reduced our problem to the case $d \varphi=A_{\lambda} X^{\lambda}+c^{\lambda} P_{\lambda}$. If the $A_{2}$ here involved only $d t^{1}, \cdots, d t^{m}$, then by 2.6 and 2.4 this $\varphi$ would be Hamiltonic.

In the contrary case, write

$$
d \varphi=a_{\lambda \ldots \tau} d x^{\lambda} \cdots d x^{\tau}+R
$$

where $a_{\lambda \ldots \tau}$ is a form involving only the $d p$ 's and $d t$ 's, where the number of indices $\lambda \cdots \tau$ is $N$, and $R$ is form wherein each term has less than $N$ factors $d x^{\mu}$. We take the $a_{2 \ldots \tau}$ to be alternating, and assume that one of them is not 0 .

Let $d_{p}, d_{t}, d_{x}$ denote exterior differentiations with respect to the $p$ 's, the $t$ 's, the $x$ 's, respectively. Of course

$$
\left(d_{p}+d_{t}+d_{x}\right)\left(a_{\lambda \ldots \tau} d x^{\lambda} \cdots d x^{\tau}+R\right)=0 .
$$

From this one can see that 


$$
d_{x}\left(a_{\lambda \ldots \tau} d x^{\lambda} \cdots d x^{\tau}\right)=0 .
$$

By Poincare's lemma we can write

$$
a_{\lambda \ldots \tau} d x^{\lambda} \cdots d x^{\tau}=d_{x} A_{\lambda \ldots o} d x^{\lambda} \cdots d x^{\sigma}
$$

in some neighborhood of the point in question. Here we may take the $A_{\lambda \ldots \sigma}$ to be alternating in their $N-1$ indices.

Let

$$
\begin{aligned}
\Phi & =A_{\lambda \cdots \rho_{\sigma}} d x^{\lambda} \cdots d x^{\rho} X^{\sigma} \\
& =A_{\lambda \cdots_{\sigma}} d x^{\lambda} \cdots d x^{\sigma}+A_{\lambda \cdots_{\tau}} d x^{\lambda} \cdots d x^{\rho} T^{\sigma}
\end{aligned}
$$

where $T^{\sigma}=X^{\sigma}-d x^{\sigma}$, a form with no $d x$. Now

$$
d \Phi=a_{\lambda \omega_{\tau}} d x^{\lambda} \cdots d x^{\tau}+d_{x}\left(A_{\lambda \cdots \rho \sigma} d x^{\lambda} \cdots d x^{\rho} T^{\sigma}\right)+\left(d_{p}+d_{t}\right) \Phi,
$$

whence $d(\varphi-\Phi)=R-d_{x}\left(A_{\lambda \ldots o} d x^{\lambda} \cdots T^{o}\right)-\left(d_{p}+d_{\tau}\right) \Phi$. The form on the right has degree less than $N$ in the $d x^{\lambda}$. By induction we may therefore assume

$$
d \varphi=X^{\lambda} A_{\lambda}+c^{\lambda} P_{\lambda}
$$

where $A_{\lambda}$ is a form involving only the $d p$ 's and $d t$ 's. We break up $A_{\lambda}$ in the manner

$$
A_{\lambda}={ }_{N} A_{\lambda}+{ }_{N-1} A_{\lambda}+\cdots+{ }_{0} A_{\lambda}
$$

where ${ }_{j} A_{\lambda}$ is homogeneous of degree $j$ in the $d p$ 's (and hence of degree $m-1-j$ in the $d t$ 's). We assume specifically that some ${ }_{N} A_{\lambda}$ is not 0 . Anyway

$$
d \varphi=d x^{\lambda}\left({ }_{N} A_{\lambda}+\cdots+{ }_{0} A_{\lambda}\right)+c^{\lambda} P_{\lambda} .
$$

For greater brevity, write

$$
d \varphi=d x^{\lambda}\left(a_{\lambda}+b_{\lambda}+\cdots\right)+c^{\lambda} P_{\lambda} .
$$

So

$$
\begin{aligned}
0= & -d x^{\lambda}\left(d_{p} a_{\lambda}+d_{t} a_{\lambda}+d_{x} a_{\lambda}+d_{p} b_{\lambda}+d_{t} b_{\lambda}+d_{x} b_{\lambda}+\cdots\right) \\
& +\left(d_{p} c^{\lambda}+d_{t} c^{\lambda}+d_{x} c^{\lambda}\right) P_{\lambda}-c_{\lambda}\left(d_{p} P_{\lambda}+d_{t} P_{\lambda}+d_{x} P_{\lambda}\right) .
\end{aligned}
$$

Now $d x^{2} d_{x} a_{\lambda}$ has $2 d x^{\prime}$ 's and nothing else does, so $d_{x}\left(d x^{\lambda} a_{\lambda}\right)=0$. Next consider that $d x^{2} d_{p} a_{\lambda}$ has $N+1 d p$ 's, and one $d x$. If $N \geqq 1$, then $d_{x} c^{\lambda} P_{\lambda}$ and $d_{x} P_{\lambda}$ haven't enough $d p$ 's to cancel $d x^{\lambda} d_{p} a_{\lambda}$. So $d_{p}\left(d x^{\lambda} a_{\lambda}\right)=0$.

Denote $d x^{\lambda} a_{\lambda}$ by $\psi$. So $\left(d_{x}+d_{p}\right) \psi=0$. I declare that there is a form $\omega$ in a neighborhood of the point in question such that $\psi=d_{x} d_{p} \omega$. This is really a general lemma with hypotheses: 
$\left(d_{p}+d_{x}\right) \psi=0$ and $\psi$ is homogeneous of degree $N \geqq 1$ in $d p$ and homogeneous of degree $M \geqq 1$ in $d x$. Locally, then,

$$
\psi=\left(d_{p}+d_{x}\right)\left({ }_{M+N-1} \omega_{0}+{ }_{M+N-2} \omega_{1}+\cdots+{ }_{0} \omega_{M+N-1}\right)
$$

where the suffixes show the degree of homogeneity in $d p, d x$ (respectively) of each term. Thus ir is the sum of the entries of the matrix

$$
\left(\begin{array}{cccc}
\delta_{M+N-1} \omega_{0} & \delta_{M+N-2} \omega_{1} \cdots & \delta_{M-1} \omega_{N} \cdots \delta_{0} \omega_{M+N-1} & 0 \\
0 & \theta_{M+N-1} \omega_{0} & \cdots & \theta_{M} \omega_{N-1}
\end{array}\right] .
$$

Here $\delta=d_{p}, \theta=d_{x}$, and the two entries of each column agree in their homogeneity. From the first column we obtain ${ }_{M+N-2} \omega_{0}=\delta b_{1}$. From the second column we see that $\delta_{M+N-2} \omega_{1}+\theta \delta b_{1}=0$. Observing $\theta \delta=-\delta \theta$ we conclude ${ }_{M+N-2} \omega_{1}-\theta b_{1}=\delta b_{2}$. We continue thus until we have dealt with the $N$ th column, from which we obtain ${ }_{M} \omega_{N-1}$ $\theta b_{N-1}=\delta b_{N}$. Then we begin at the other end, and work backwards, introducing forms $c_{J}$. The final result can be guessed by interchanging $M$ and $N, \delta$ and $\theta:_{M-1} \omega_{N}-\delta c_{M-1}=\theta c_{M}$. Now we observe that $\psi$ is the sum of the $(N+1)$ th column:

$$
\begin{aligned}
\psi^{\prime} & =\delta_{M-1} \omega_{N}+\theta_{M} \omega_{N-1} \\
& =\delta\left(\delta c_{M-1}+\theta c_{M}\right)+\theta\left(\theta b_{N-1}-\delta b_{N}\right) \\
& =\delta \theta\left(c_{M}+b_{N}\right) .
\end{aligned}
$$

Thus in our particular case

$$
\psi=d_{x} d_{p} \omega=d x^{\lambda} \frac{\partial}{\partial x^{\lambda}} d_{p} \omega
$$

where $\partial / \partial x$ of any form means simply differentiate each coefficient. The result is

$$
a_{\lambda}=\frac{\partial}{\partial x^{\lambda}} d_{p} \omega
$$

Define

$$
\omega_{\lambda}=\frac{\partial}{\partial x^{\lambda}} \omega
$$

and let $\Phi=X^{2} \omega_{\lambda}$. Recall $T^{o}$. Then

$$
\begin{aligned}
d \Phi= & \left(d X^{\lambda}\right) \omega_{\lambda}+X^{\lambda}\left(d_{p} \omega_{\lambda}+d_{t} \omega_{\lambda}+d_{x} \omega_{\lambda}\right) \\
= & d\left(-T^{\lambda}\right) \omega_{\lambda}+d x^{\lambda}\left(d_{t} \omega_{\lambda}+d x^{\mu} \frac{\partial^{2} \omega_{\lambda}}{\partial x^{\mu} \partial x^{\lambda}}\right) \\
& +X^{\lambda} a_{\lambda}-T^{\lambda}\left(d_{t} \omega_{\lambda}+d x^{\mu} \frac{\partial^{2} \omega_{\lambda}}{\partial x^{\mu} \partial x^{\lambda}}\right) .
\end{aligned}
$$


Therefore

$$
\begin{aligned}
d(\Phi-\varphi)= & d\left(-T^{\lambda}\right) \omega_{\lambda}+d x^{\lambda}\left(d_{t} \omega_{\lambda}\right) \\
& -T^{\lambda}\left(d_{t} \omega_{\lambda}+d x^{\mu} \frac{\partial^{2} \omega_{\lambda}}{\partial x^{\mu} \partial x^{\lambda}}\right)-c^{\lambda} P_{\lambda} .
\end{aligned}
$$

We must now examine the $d x$ terms on the right and see that their degree in $d p$ is less than $N$. Here we keep in mind that $\omega$ is of degree $N-1$. Thus we can arrive at a "new" $\varphi$ with

$$
d \varphi=X^{\lambda} A_{\lambda}+c^{\lambda} P_{\lambda}
$$

where $A_{\lambda}$ has no $d p$. As already remarked, this ultimate $\varphi$ is Hamiltonic. Thus ends our proof of 3.2.

4. Changes of coordinates. We show next that changes in the coordinates for $Q$ used in 3.1 leave unchanged the manifestly dynamic forms.

If we choose new coordinates $y^{3}, \cdots, y^{n}$ in $Q$ and adopt them in $J^{(1)}\left(Q, R^{m}\right)$ then we have even there

$$
d y^{\hat{\xi}}=\frac{\partial y^{\hat{\xi}}}{\partial x^{\lambda}} d x^{\lambda}
$$

For the "momenta" associated to the $y^{\xi}$, which may be called $q_{\hat{\kappa}}^{i}$, the chain rule gives

$$
q_{\xi}^{i}=p_{\lambda}^{i} \frac{\partial x^{\lambda}}{\partial y^{\xi}}
$$

whence the simple relation

$$
\frac{\partial}{\partial q_{\xi}^{i}}=\frac{\partial y^{\xi}}{\partial x^{\lambda}} \frac{\partial}{\partial p_{\lambda}^{i}}
$$

The chain rule also gives

$$
\frac{\partial}{\partial y^{\xi}}=\frac{\partial x^{\lambda}}{\partial y^{\xi}} \frac{\partial}{\partial x^{\lambda}}-\frac{\partial y^{\eta}}{\partial x^{\lambda}} S_{\xi \eta}^{i} \frac{\partial}{\partial p_{\lambda}^{i}}
$$

where

$$
S_{\xi \eta}^{i}=\frac{\partial^{2} x^{\mu}}{\partial y^{\xi} \partial y^{\eta}} p_{\mu}^{i}
$$

As a result

$$
\alpha=E_{i j, \cdots, k} q_{\xi}^{i} d y^{\xi} d t^{j} \cdots d t^{k}-H d t^{1} \cdots d t^{k} .
$$


Proposition.

$$
Y^{\xi}=\frac{\partial y^{\xi}}{\partial x^{\lambda}} X^{\lambda}
$$

We give a proof for $m=3$. Taking $i=1$ in 2.4 gives

$$
\left.\frac{\partial}{\partial p_{\lambda}^{1}}\right\rfloor d \alpha=\left(d \alpha^{\lambda}-\frac{\partial H}{\partial p_{\lambda}^{1}} d t^{1}\right) d t^{2} d t^{3}
$$

From this you get at once that

$$
\left.\left.X_{1}^{\lambda} \equiv \frac{\partial}{\partial t^{3}}\right\lrcorner \frac{\partial}{\partial t^{2}}\right\lrcorner \frac{\partial}{\partial p_{\lambda}^{1}}=d x^{\lambda}-\frac{\partial H}{\partial p_{\lambda}^{1}} d t^{1} .
$$

Taking also $i=2$ or 3 gives

$$
\left.\left.\left.\left.J_{i}^{\lambda} \equiv \frac{\partial}{\partial t^{3}}\right\rfloor \frac{\partial}{\partial t^{2}}\right\rfloor \frac{\partial}{\partial t^{1}}\right\rfloor \frac{\partial}{\partial p_{\lambda}^{1}}\right\rfloor d \alpha=-\frac{\partial H}{\partial p_{\lambda}^{i}} .
$$

Now

$$
X^{\lambda}=X_{1}^{\lambda}-J_{2}^{\lambda} d t^{2}-J_{3}^{\lambda} d t^{3} .
$$

From here we get an expression for $X^{\lambda}$ in terms of the $\partial / \partial p$ in which these $\partial / \partial p$ are the only things that change, and from which we can see that 4.1 implies 4.2 .

THEOREM 4.3. The class of manifestly dynamic forms is the same for any two coordinate systems.

Proof. Say $\varphi$ is manifestly dynamic in the $x$ coordinates. Then $\varphi-F_{\lambda} X^{\lambda}$ is Hamiltonic. From 4.2 one can obtain $G_{\mu}$ such that $\varphi-G_{\mu} Y^{\mu}$ is the same Hamiltonic form. Hence $\varphi$ is manifestly dynamic in the $y$ coordinates.

We note the following, to emphasize in difference from 4.2:

$$
Q_{\hat{\xi}}=\frac{\partial x^{\lambda}}{\partial y^{\xi}} P_{\lambda}+\frac{\partial y^{\eta}}{\partial x^{\lambda}} S_{\xi \eta}^{i} X^{\lambda} E_{i j \ldots k} d t^{j} \cdots d t^{k} .
$$

5. A global version of Theorem 3.1. A grobal version of 3.1 would follow from 3.1 if one could assert that the $F_{\lambda} X^{\lambda}$ computed in one coordinate neighborhood to make $\varphi-F_{\lambda} X^{\lambda}$ Hamiltonic had to be the same as the $G_{\mu} Y^{\mu}$ computed in some other neighborhood (on the overlap, of course).

We surely could assert this if the following were true.

5.1. $H$ is such that when $\varphi$ is of the form $F_{\lambda} X^{\lambda}$ and $d \varphi$ is of 
the form $U\rfloor d \alpha$, then $\varphi$ must be 0 .

For $m=2, n=1$ we know precisely for which $H$ (5.1) holds. In more general cases we can give examples where it holds.

Before examining when 5.1 holds we will show that it certainly does not always hold. We will deal with the case $m=2$ and $Q=\boldsymbol{R}$. So $n=1$. We abbreviate $p_{1}^{1}$ by $p, p_{1}^{2}$ by $q, t^{1}$ by $s, t^{2}$ by $t$ and so

$$
\alpha=p d x d t+q d s d x-H d s d t .
$$

THEOREM 5.3. Let $H$ be analytic and satisfy the four partial differential equations

$$
H_{p p}=H_{p q}=H_{q q}=H_{q s}-H_{p t}+H_{p} H_{q x}-H_{q} H_{p x}=0 .
$$

Then every dynamic form is Hamiltonic.

Sketch of proof. With these hypotheses, the $H$ is linear in $p$, $q$, and

$$
H=a(s, t, x) p+b(s, t, x) q+c(s, t, x)
$$

where $a, b, c$ satisfy the partial differential equation

$$
b_{s}-a_{t}+a b_{x}-b a_{x}=0 \text {. }
$$

I now claim

5.5. Let $P, Q, R, S$ be four numbers such that

$$
P+Q+a_{x} p+b_{x} q+c_{x}=0
$$

at some point $T_{0}$ of $J^{1}\left(\boldsymbol{R}, \boldsymbol{R}^{2}\right)$. Then there is an extremal $p=p(s, t)$, $q=q(s, t), x=x(s, t)$ which passes through $T_{0}$ and makes hold at $T_{0}: p_{s}=P, q_{t}=Q, p_{t}=S, q_{s}=R$.

Proof. It will suffice to find only those motions wherein $x, p, q$, are expressed in terms of $s, t$. In such cases, the relevant differential equations are just the Euler equations for

$$
\delta \iint\left[p \frac{\partial x}{\partial s}+q \frac{\partial x}{\partial t}-H\right] d s d t=0
$$

namely

$$
\begin{aligned}
\frac{\partial x}{\partial s}-\frac{\partial H}{\partial p} & =0 \\
\text { (q) }-\frac{\partial x}{\partial t}-\frac{\partial H}{\partial q} & =0
\end{aligned}
$$


$(x)$

$$
\frac{\partial}{\partial s} p+\frac{\partial}{\partial t} q=-\frac{\partial H}{\partial x}
$$

In the present notation these are

$$
\frac{\partial x}{\partial s}=a, \quad \frac{\partial x}{\partial t}=b
$$

and (using subscripts for derivatives)

$$
p_{s}+q_{t}+a_{x} p+b_{x} q+c_{x}=0 .
$$

Let $T_{0}$ have coordinates $\left(p_{0}, q_{0}, s_{0}, t_{0}, x_{0}\right)$. The integrability conditions for the first two equations are precisely (5.4). Thus we can find $x$ with $x\left(s_{0}, t_{0}\right)=x_{0}$.

We replace all $x$ 's in (5.6) by our solution $x(s, t)$. We replace $q$ in (5.6) by $q_{0}+R s+Q t$. Now we solve the resulting equation for $p$ using the initial data $p=p_{0}+S t$ for $s=0$. Thus (5.4) is proved, and we have found a 3-parameter family of motions.

We continue to describe our proof of the theorem. We look for all pairs $U, V$ of vectors at $T_{0}$ tangent to these motions. These pairs form a 3-parameter family. We now calculate conditions on an arbitrary 2-form $\Phi$ that $\langle\Phi ; U, V\rangle=0$ for such pairs $U, V$. We also calculate the condition that $\Phi$ have a representation as $\left.F_{0}\right\lrcorner(d \alpha)_{T_{0}}$ with some vector $F_{0}$ at $T_{0}$. We find that these conditions coincide.

If $\varphi$ is dynamic, let $\Phi=d \varphi$. At $T_{0}$ it has the form $\left.F_{0}\right\rfloor(d \alpha)_{T_{0}}$. Varying $T_{0}$ gives us a vector field $F$ such that $\left.d \varphi=F\right\rfloor(d \alpha)$. The vector field $F$ is smooth because, $m$ being greater than 1 , it is unique. This establishes (5.3).

When $m=2$ and $n=1$, the four quantities $H_{p p}, H_{p q}, H_{q q}, H_{q s}-$ $H_{p t}+H_{p} H_{q x}-H_{q} H_{p x}$ mentioned in (5.3) lie at the heart of problem (5.1). If these four quantities have no common zeros, then (5.1) holds.

We omit the proof of this statement. The proof is easy. However, its generalization to other $m, n$ is not known.

We can prove weakened versions of this statement. We prepare the notation for presenting one such weakened version.

Let $v^{\lambda}, p_{\lambda}^{i}, t^{i}$ be coordinates as discussed in $\S 2$ and let $H$ be the function $H$ appearing in the given $m$-form $\alpha$. Define

$$
H_{i j}^{\lambda \mu^{\prime \prime}}=\frac{\partial^{2} H}{\partial p_{\lambda}^{i} \partial p_{\mu}^{j}}
$$

for a fixed $i$ and $j(1 \leqq i, j \leqq m)$ we have an $n \times n$ matrix $H_{i j}$ whose entries are these $H_{i j}^{\lambda \mu}$.

Let $T$ be a point of this coordinate neighborhood. We will say that condition $H$ holds at $T$ if for each index $i$ there exist numbers 
$c_{1}, \cdots, c_{m}$ and integers $k_{1}, \cdots, k_{m}$ such that $\sum_{j=1}^{m} c_{j} H_{i j}$ is 0 while $\sum_{j=1}^{m} c_{j} H_{j k_{j}}$ is invertible.

We will say that an $m$-form $\alpha$ of the sort considered on $J^{(1)}\left(Q, R^{m}\right)$ satisfies condition $H$ if at each point there is a coordinate system such that $H$ holds.

THEOREM 5.7. If $\alpha$ satisfies condition $H$, then (5.1) holds.

We judge that a proof just with $m=3$ will adequately indicate the method of proof for the general case.

We start the proof by pointing out that if $\varphi$ is a 2-form expressible as $F_{\lambda} X^{\lambda}$ then

$$
\Phi=a_{\lambda \mu} X^{\lambda} X^{\mu}+b_{i \lambda}^{\prime \prime} d p_{\mu}^{i} X^{\lambda}+c_{i \lambda} d t^{i} X^{\lambda},
$$

and

$$
\begin{aligned}
d \varphi= & d a_{\lambda_{i}^{\mu}} X^{\lambda} X^{\mu}-2 a_{\lambda_{\mu}} d H_{i}^{\lambda} d t^{i} X^{\mu} \\
& +d b_{i \lambda}^{\mu} d p_{\lambda}^{i} X^{\lambda}+d c_{i \lambda} d t^{i} X^{\lambda}+c_{i \lambda} d t^{i} d H_{j}^{\lambda} d t^{j} \\
& +b_{i \lambda}^{\mu} d p_{\mu}^{i} H_{j}^{\lambda} d t^{j} .
\end{aligned}
$$

Here the order is not quite the natural one and is intended to exhibit as the last term the term which shall be discussed first. This term is a sum of several terms, of which one is

$$
b_{i \lambda}^{\mu} H_{j k}^{\lambda \nu} d p_{\mu}^{i} d p_{\nu}^{k} d t^{j}
$$

and one can see that this is the only term in $d \varphi$ of the type $d p d p d t$. We will now show that $U\lrcorner d \alpha$ has no term of the type $d p d p d t$, so that (5.8) must vanish. Recall that

$$
d \alpha=-X^{\lambda} P_{\lambda}=-X^{\lambda}\left(E_{i j k} d p_{\lambda}^{i} d t^{j} d t^{k}+H_{x^{\lambda}} d t^{1} d t^{2} d t^{3}\right) .
$$

Let $U$ be a vector field. Let $\left\langle U, d t^{i}\right\rangle=U^{i},\left\langle U, d p_{\lambda}^{i}\right\rangle=U_{\lambda}^{i}$, and let $\left\langle U, X^{\lambda}\right\rangle=V^{\lambda}$. Then

$$
\begin{array}{r}
U\rfloor d \alpha=-V^{\lambda} P_{\lambda}+X^{\lambda}\left\{E_{i j k}\left(U_{\lambda}^{i} d t^{j} d t^{k}+2 U^{j} d p_{\lambda}^{i} d t^{k}\right)\right. \\
+H_{x^{2}}\left(U^{1} d t^{2} d t^{3}-U^{2} d t^{1} d t^{3}+U^{3} d t^{1} d t^{3}\right) .
\end{array}
$$

Indeed, there are no $d p d p d t$ terms, so (5.8) is 0 . Consequently

$$
b_{i \lambda}^{\mu} H_{j k}^{\lambda \nu}-b_{k \lambda}^{\nu} H_{j i}^{\lambda \mu}=0 \text {. }
$$

Let $k=k_{j}$, multiply by the $c_{j}$ given by hypothesis, and sum on $j$. Therefore

$$
b_{i \lambda}^{\prime \prime} \sum_{j} c_{j} H_{j k_{j}}^{\lambda_{\nu}}=b_{i \lambda}^{\prime \prime} K^{\lambda \nu}=0
$$

where $K$ is invertible. Thus the $b_{i \lambda}^{\mu}$ are all 0 . 
We now look for $d p d t d t$ terms in $d \varphi$, and equate with those in $U\lrcorner d \alpha$. This yields

$$
c_{i \lambda} d t^{i} H_{j k}^{\lambda \mu} d p_{\mu}^{k} d t^{j}=-V^{\lambda} E_{i j_{k}} d p_{\lambda}^{i} d t^{j} d t^{k} .
$$

From this

$$
-c_{i \lambda} H_{j k}^{\lambda \mu}+c_{j \lambda} H_{i k}^{\lambda \mu}=-2 V^{\mu} E_{i j k} .
$$

We sum the values of this for $(i j k)=(123),(231),(312)$. The left hand sides yield 0 and the right hand side $-12 V^{\mu}$, because $E_{123}=2$. So the $V$ 's are 0 . Using our hypothesis as before, we obtain $c_{i \lambda}=0$. We examine the remainder of $d \varphi$ for $d p d t X$ terms, and equate. This yields

$$
-2 a_{\lambda \mu} H_{i j}^{\lambda_{\nu}} d p_{\nu}^{j} d t^{i} X^{\mu}=2 X^{\lambda} E_{i j k} U^{j} d p_{\lambda}^{i} d t^{k} .
$$

For the components one obtains

$$
a_{\lambda \mu} H_{i k}^{\lambda \nu}=-E_{k j i} U^{j} \delta_{\mu}^{\nu} .
$$

Pick $\mu$. Then there exists a $\nu \neq \mu$ unless $n=1$ (and then there would be no $\left.a_{\lambda \mu} !\right)$. So

$$
a_{\lambda \mu} H_{i k}^{\lambda_{\nu}}=0
$$

and

$$
a_{\lambda \mu} \sum c_{i} H_{i k_{i}}^{\lambda \nu}=0 \text {. }
$$

This forces $a_{\lambda \mu}=0$ for all $\lambda$. Thus $\varphi=0$.

An easy way to obtain condition $H$ is to let $H$ be the sum of the squares of all the $p_{\lambda}^{i}$.

Let me repeat that I don't think condition $H$ has any deep connection with (5.1). It just happens to be a condition which is rather easy to satisfy, and through (5.7) shows that (5.1) is a reasonable way to obtain a global version of (3.2), as follows:

THEOREM 5.9. Suppose (5.1) holds. Let $\varphi$ be a manifestly dynamic form. Then there exists a form $\psi$ which in each coordinate system can be expressed as $F_{\lambda} X^{\lambda}$ such that $\phi-\psi$ is Hamiltonic.

We repeat, forms like this $\psi$ vanish on all motions.

6. Are all dynamic forms manifestly so? When $m=2$ and $n=1$, all dynamic forms are manifestly so. To show this, we use a Theorem 6.1 presented below. We mention its generalization to general $m, n$ in (6.2). However, (6.2) does not enable us to prove that all dynamic forms are manifestly so for all $m, n$. In fact, this 
is false for $m=3, n=1$ and $m=2, n=2$. Examples are given below.

Let us use the notation of (5.2) and (5.3).

Theorem 6.1. Let $\left(p_{0}, q_{0}, 0,0, x_{0}\right)$ be a point $T$ of $J^{(1)}\left(\boldsymbol{R}, \boldsymbol{R}^{2}\right)$. Suppose that the rank of the Hessian

$$
\left(\begin{array}{ll}
H_{p p} & H_{p q} \\
H_{p q} & H_{q q}
\end{array}\right)
$$

is at least 1 at $T$. Suppose $H$ is analytic. Let $U_{1}$ and $U_{2}$ be two vectors at $T$, with $p, q, s, t, x$ components as given in the array

$$
\left(\begin{array}{llllr}
a & b & 1 & 0 & c \\
u & v & 0 & 1 & w
\end{array}\right)
$$

Suppose $X, d X, P$ vanish on the subspace of the tangent space $T^{1}(K)$ at $T$. Then there is an extremal submanifold which is tangent to $T_{1}$ and $T_{2}$.

Proof. By a rotation in the $s-t$ plane we can arrive at the case where $H_{p p} \neq 0$ at $T$. The hypotheses now yield that $c=H_{p}$, $w=H_{q}, a+v+H_{x}=0$, and

$$
H_{p p}(-u)+H_{p q}(-v+a)+H_{q q} b+H_{q s}-H_{p t}-H_{p x} w+H_{q x} c=0,
$$

are true at $T$.

Let $p(s)=p_{0}+a s, a(s)=q_{0}+b s$, and solve the system

$$
\begin{gathered}
\frac{d x}{d s}=H_{p}\left(p_{0}+a s, q_{0}+b s, s, 0, x\right), \\
x(0)=x_{0} .
\end{gathered}
$$

According to $[10, \S 7]$, we can pose a Cauchy problem with $p(s)$, $q(s), x(s)$ as the data for $t=0$, and

$$
\frac{\partial x}{\partial s}=H_{p}, \frac{\partial x}{\partial t}=H_{q}, \frac{\partial p}{\partial s}+\frac{\partial q}{\partial t}=-H_{x}
$$

as the differential equations. Because of the analyticity, we can solve this system. As in (5.5), if (6.14) is solved, then the submanifold $p=p(s, t), q=q(s, t), x=x(s, t)$ is extremal. Let us call it $A$.

Now we show that $U_{1}$ is tangent to $A$. Let $s$ increase by $\varepsilon$, but keep $t=0$. Then approximately $p$ increases by $a \varepsilon, q$ by $b \varepsilon$, and $x$ by $H_{p} \varepsilon=c \varepsilon$. Thus the tangent for the section $t=0$ is the given $U_{1}$. 
Next, keep $s=0$ and let $t$ grow. Then $x$ grows by $H_{q} \varepsilon=w \varepsilon$, $q$ grows by $\left(-H_{x}-a\right) \varepsilon=v \varepsilon$. More precisely, $\partial x / \partial t=H_{q}, \partial q / \partial t=v$, $\partial p / \partial s=a, \partial q / \partial s=b$ and $a+v+H_{x}=0$.

One must now write down in full the consequences of

$$
\frac{\partial}{\partial t} H_{p}=\frac{\partial}{\partial s} H_{q}
$$

The result resembles (6.13) to such an extent that it, together with (6.13), forces $\partial p / \partial t=u$. Thus $U_{2}$ is tangent to $A$ and the proof of (6.6) is complete.

The generalization of (6.1) for general $m, n$ may be of interest.

THEOREM 6.2. Let $T$ be a point of $J^{(1)}\left(Q, R^{m}\right)$. Let $U_{1}, \cdots, U_{m}$ be vectors at $T$ such that the $t^{i}$ component of $U_{j}$ is $\delta_{j}^{i}$. Suppose that the forms $X^{\lambda}, d X^{\lambda}$, and $P_{\lambda}$ all vanish on the linear space spanned by these $U_{j}$ at $T$. Suppose also that the rank of the matrix

$$
\frac{\partial^{2} H}{\partial p_{\lambda}^{i} \partial p_{\mu}^{j}}
$$

is at least $n(m-1)$ where $\operatorname{dim} Q=n$. Suppose $H$ is analytic. Then there is an extremal for (1.1) to which $U_{1}, \cdots, U_{m}$ are tangent.

CoROLLARY 6.3. Let $\mu$ be an $m$-form in $J^{(1)}\left(Q, R^{m}\right)$ which vanishes on all motions in the sense that $\mu \mid E=0$ for each motion $E$. Let the hypotheses of (6.2) concerning $T, U_{1}, \cdots, U_{m}$ and $H$ hold. Then $\left\langle\mu ; U_{1}, \cdots, U_{m}\right\rangle=0$.

This is obviously a consequence of (6.2).

THEOREM 6.4. Consider an $\alpha$ as in (5.2), where $H$ is as in (6.1). Then every 2-form which vanishes on all motions can be expressed as

$$
\mu=A X+b d X+c P
$$

where $A$ is $a$ 1-form, $b$ and $c$ are scalars, while $X$ and $P$ are the forms (2.1), (2.2) $(m=2, n=1)$.

Proof. As in the proof of (6.1), we can easily bring it about that $H_{p p} \neq 0$.

If $\mu$ and $\mu^{\prime}$ are two forms such that $\mu-\mu^{\prime}$ can be written as $A X+b d X+c P$ we will write $\mu \sim \mu^{\prime}$.

If $\mu \sim \mu^{\prime}$ and $\mu$ vanishes on all motions then so does $\mu^{\prime}$.

From $P=d q d s-d p d t-H_{x} d s d t$ we see that $d p d t \sim a$ form 
with only $d q d s$ and $d s d t$. Let us use $d p, d q, d s, s t$, and $X$ itself as a basis for forms. Now $X \sim 0$. So given any $\mu$, we can write $\mu \sim \mu^{\prime}$ where $X$ and $d p d t$ do not appear in $\mu^{\prime}$. More can be said by examining $d X . \quad d X$ contains $-H_{p p} d p d s$. Therefore $d p d s \sim a$ form with no $X$, no $d p d t$. Hence we may assume that $\mu^{\prime}$ has no $X$, no $d p d s$, and no $d p d t$.

Therefore assume

$$
\mu=\mu_{p q} d p d q+\mu_{q s} d q d s+\mu_{q t} d q d t+\mu_{s t} d s d t .
$$

As (6.3) suggests, consider $U_{1}$ and $U_{2}$ with $d p, d q, d s$, $d t$ components given by the rows of

$$
\begin{array}{llll}
a & b & 1 & 0 \\
c & d & 0 & 1,
\end{array}
$$

and $X$ components. The relation $\left\langle P ; U_{1}, U_{2}\right\rangle=0$ imposes $-d-a$ $-H_{x}=0$. We calculate $d X$ and replace $d x$ by $X+H_{p} d s+H_{q} d t$, obtaining

$$
\begin{gathered}
d X=H_{p p} d p d s+H_{p q} d q d s+H_{q p} d p d t+H_{q q} d q d t \\
+J X+k d s d t .
\end{gathered}
$$

Therefore

$$
\left\langle d X ; U_{1}, U_{2}\right\rangle=H_{p p}(-c)+H_{p q}(-d)+H_{q p} a+H_{q q} b+k,
$$

which is supposed to be 0 , when the previous relation and

$$
\left\langle\mu ; U_{1}, U_{2}\right\rangle=\mu_{p q}(a d-b c)+\mu_{q s}(-d)+\mu_{q t} b+\mu_{s t},
$$

hold. From this the reader can easily deduce that $\mu=0$.

We now come to the counterexample. The first one is in $J^{(1)}\left(\boldsymbol{R}^{3}, \boldsymbol{R}\right)$ and is based on the following

Proposition 6.5. Let $H=\frac{1}{2}\left[\left(p^{1}\right)^{2}+\left(p^{2}\right)^{2}+\left(p^{3}\right)^{2}\right]$. Then a 3 -form vanishes ${ }^{1}$ on all the motions if and only if it is of the type

$$
A X+B d X+c P+f d \varphi
$$

where

$$
\varphi=p^{3}\left(d p^{1} d t^{1}-d p^{2} d t^{2}\right),
$$

and $A, B, c, f$ are forms of appropriate degree.

The proof is very routine and we leave it to the reader. Of course (6.5) implies that $\phi$ is dynamic. The counterexample is

${ }^{1}$ By vanishes on a motion $E$ we mean the restriction to $E$ is 0 . 
provided by the following:

Proposition 6.6. $\varphi$ is not manifestly dynamic.

We omit the proof here also.

We now turn to $J^{(1)}\left(\boldsymbol{R}^{2}, \boldsymbol{R}^{2}\right)$.

Proposition 6.7. Let $H=\frac{1}{2}\left[\left(p_{1}^{1}\right)^{2}+\left(p_{1}^{2}\right)^{2}+\left(p_{2}^{1}\right)^{2}+\left(p_{2}^{2}\right)^{2}\right]$. Then a 2-form vanishes on all the motions if and only if it is of the type

$$
A_{1} X^{1}+A_{2} X^{2}+B_{1} d X^{1}+B_{2} d X^{2}+c^{1} P_{1}+c^{2} P_{2}+f d \varphi+g d \psi
$$

where $A_{1}, \cdots, g$ are forms of appropriate degree and

$$
\varphi=p_{1}^{1} d p_{2}^{1}-p_{1}^{2} d p_{2}^{2}, \quad \psi=p_{1}^{1} d p_{2}^{2}-p_{1}^{2} p_{2}^{1} .
$$

The counterexample consists in the observation, which is easily proved, that $\varphi$ and $\psi$ here are not manifestly dynamic.

There is an observation about the $\alpha$ (and $H$ ) in (6.5) which is relevant to a question not yet raised in this paper. The observation is as follows.

Proposition 6.8. A 2-form vanishes on all the motions if and only if it is of the type

$$
A X+b d X
$$

where $A$ and $b$ are forms of appropriate degree (one less than in (6.5)).

Such a form is manifestly dynamic ${ }^{2}$. We have therefore the following, which destroys a natural conjecture.

THEOREM 6.9. There exists a dynamic form $\rho$ for which there cannot be found a Hamiltonic $\varphi^{\prime}$ such that $\varphi-\varphi^{\prime}$ vanishes on every motion.

The $\varphi$ we have in mind here is that of (6.5).

7. Transformation theory. It seems fair to say that the "transformation theory" of classical dynamics [9] is concerned with choosing new coordinates so that $\alpha=p_{i} d x^{i}-H d s$ takes the form

$$
\alpha=P_{i} d X^{i}-\widetilde{H} d S
$$

\footnotetext{
2 This assertion concerns only the example of (6.5).
} 
where $\tilde{H}$ is constant, as promised by Darboux' theorem.

It is easy to see that such a transformation theory is not possible when $m \geqq 2$.

THEOREM 7.1. Suppose $H$ is as in (5.7). Suppose also $H$ is analytic. Then it is not possible to find coordinates $P, Q, S, T, X$ in $K$ and a function $Z$ on $K$ such that (see (5.2))

$$
\alpha=P d X d T+Q d S d X-Z d S d T
$$

where $Z$ is 0 , or even where merely

$$
Z_{P P}=Z_{P Q}=Z_{Q Q}=Z_{Q S}-Z_{P T}+Z_{P} Z_{Q X}-Z_{Q} Z_{P X}=0 .
$$

Proof. If $\alpha$ had the form (7.2), then, by (5.3) every dynamic from would be Hamiltonian, whereas if $H$ is as (5.7) this would not be true.

Lest one be tempted to think that if two Hamiltonians both satisfy the hypothesis of (5.7) then their 2-forms differ only up to a change of coordinates, we offer the following.

THeOREM 7.3. Suppose $H=p H_{p}+q H_{q}$. Then one cannot find $P, \cdots, X$ and $Z$ such that (10.2) holds but $Z \neq P Z_{P}+Q Z_{Q}$.

Proof. Let $F$ have $p, q, s, t, x$ components $a, b, u, v, w$. Then a calculation shows that

$$
\begin{aligned}
\alpha \wedge(\boldsymbol{F}\lrcorner d \alpha) & \\
= & d q d s d t d x\left[\left(H-q H_{q}\right) a+p H_{q} b-p w\right] \\
& \quad-d p d s d t d x\left[q H_{p} a+\left(H-p H_{p}\right) b-q w\right] .
\end{aligned}
$$

The rank of the matrix

$$
\left(\begin{array}{cc}
H-q H_{q} & p H_{q}-p \\
q H_{p} & H-p H_{p}-q
\end{array}\right)
$$

is therefore invariant under changes of variables. This rank is 1 if and only if $H=p H_{p}+q H_{q}$. This suffices to prove (7.3).

ACKNOWLEDGMENT. This research was supported in part by the National Science Foundation, Grant MCS 78-01476.

I also gratefully acknowledge very helpful suggestions by $\mathrm{Mr}$. Ross Urwin.

\section{REFERENCES}

1. P. Dedecker, Calcul des variations, formes différentielles et champs geodésiques, coll. internat. du C.N.R.S. Strassbourg, 1953. 
2. P. L. Garcia, The Poincare-Cartan invariant in the calculus of variations, Sympos. Math., 14 (1974), 219-246.

3. K. Gawedzki, On the geometrization of the canonical formalism in classical field theory, Rep. Math. Phys., 3 (1972), 307-326.

4. Hubert Goldschmidt and Shlomo Sternberg, The Hamilton-Cartan formalism in the calculus of variations, Ann. Inst. Fourier, Grenoble 23, 1, (1973), 203-267.

5. Victor Guillemin and Shlomo Sternberg, Geometric asymtotics, Amer. Math. Soc., (1977).

6. R. Hermann, Lie algebras and quantum mechanics, Benjamin Lecture Notes, 1970.

7. J. Kijowski, A finite dimensional canonical formalism in classical field theory, Commun. math. phys., 30 (1973), 99-128.

8. J. Kijowski and W. Szczyrba, A canonical structure for classical field theories, Commun. math. phys., 46 (1976), 183-203.

9. E. T. Whittaker, Analytical Dynamics of Particles and Rigid Bodies, Cambridge U. Press, 1937.

10. Richard Arens, Reducing the order of a Lagrangian, Pacific J. Math., (1981).

Received January 12, 1979, and in revised form September 27, 1980.

UNIVERSity of CALIFORNiA

Los Angeles, CA 90024 


\section{PACIFIC JOURNAL OF MATHEMATICS}

\section{EDITORS}

DONALD BABBITT (Managing Editor)

University of Galifornia

Los Angeles, California 90024

HUgo RossI

University of Utah

Salt Lake City, UT 84112

C. C. MOORE AND ANDREW OGG

University of California

Berkeley, CA 94720

\section{J. DUGUNDJI}

Department of Mathematics University of Southern California Los Angeles, California 90007

R. Finn and J. Milgram

Stanford University

Stanford, California 94305

\section{ASSOCIATE EDITORS}
R. ARENS
E. F. BECKENBACH
B. H. NeUManN
F. WOLF
K. YosHIDA

\section{SUPPORTING INSTITUTIONS}

UNIVERSITY OF ARIZONA

UNIVERSITY OF BRITISH COLUMBIA

CALIFORNIA INSTITUTE OF TECHNOLOGY

UNIVERSITY OF CALIFORNIA

MONTANA STATE UNIVERSITY

UNIVERSITY OF NEVADA, RENO

NEW MEXICO STATE UNIVERSITY

OREGON STATE UNIVERSITY
UNIVERSITY OF OREGON UNIVERSITY OF SOUTHERN CALIFONIA STANFORD UNIVERSITY UNIVERSITY OF HAWAII UNIVERSITY OF TOKYO UNIVERSITY OF UTAH WASHINGTON STATE UNIVERSITY UNIVERSITY OF WASHINGTON 


\section{Pacific Journal of Mathematics}

\section{Vol. 93, No. $1 \quad$ March, 1981}

Richard Arens, Reducing the order of a Lagrangian $\ldots \ldots \ldots \ldots \ldots \ldots \ldots$

Richard Arens, Manifestly dynamic forms in the Cartan-Hamilton treatment

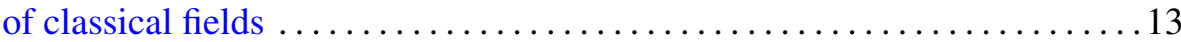

Jimmy T. Arnold, Power series rings over discrete valuation rings $\ldots \ldots \ldots 31$

Charles A. Asmuth and Joe Repka, Supercuspidal components of the

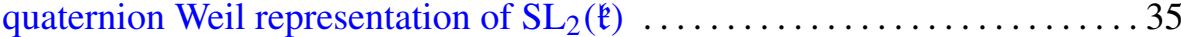

Luis A. Caffarelli and Avner Friedman, Sequential testing of several simple hypotheses for a diffusion process and the corresponding free boundary problem ................................. 49

William B. Jacob, Fans, real valuations, and hereditarily-Pythagorean fields .............................................. 95

W. J. Kim, Asymptotic properties of nonoscillatory solutions of higher order

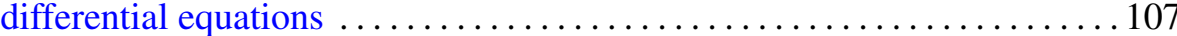

Wayne Steven Lewis, Embeddings of the pseudo-arc in $E^{2} \ldots \ldots \ldots \ldots \ldots 115$

Daniel Alan Marcus, Closed factors of normal Z-semimodules . ......... 121

Mitsuru Nakai and Leo Sario, Harmonic functionals on open Riemann surfaces ............................................. 147

John Currie Quigg, Jr., On the irreducibility of an induced

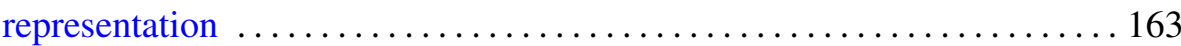

John Henry Reinoehl, Lie algebras and Hopf algebras 181

Joe Repka, Base change for tempered irreducible representations of $\mathrm{GL}(n, \mathbf{R})$

Peter John Rowley, Solubility of finite groups admitting a fixed-point-free automorphism of order $r s t$. I . . . . . . . . . . . . . . . . . . 201

Alan C. Woods, The asymmetric product of three homogeneous linear forms 